\title{
Generation of surface polaritons in dielectric cylindrical waveguides
}

\author{
A. S. Kotanjyan, ${ }^{1}$ A. R. Mkrtchyan, ${ }^{2,3}$ A. A. Saharian, ${ }^{1,4,}{ }^{*}$ and V. Kh. Kotanjyan ${ }^{4}$ \\ ${ }^{1}$ Department of Physics, Yerevan State University, 1 Alex Manoogian Street, 0025 Yerevan, Armenia \\ ${ }^{2}$ Tomsk Polytechnic University, 30 Lenin Avenue, 634050 Tomsk, Russia \\ ${ }^{3}$ Belgorod National Research University, 85 Pobedy Street, 308015 Belgorod, Russia \\ ${ }^{4}$ Institute of Applied Problems in Physics, 25 Nersessian Street, 0014 Yerevan, Armenia
}

(Received 2 February 2019; published 11 April 2019)

\begin{abstract}
We investigate the radiation of surface polaritons by a charged particle rotating around a dielectric waveguide. The general case is considered when the waveguide is immersed in a homogeneous medium. For the evaluation of the corresponding electromagnetic fields the electromagnetic Green tensor is used. A formula is derived for the spectral distribution of the radiation intensity for surface-type modes. It is shown that the corresponding waves are radiated on the eigenmodes of the dielectric cylinder. We demonstrate that the number of radiated quanta for surface polaritons of a given harmonic can be essentially larger than that for guiding modes of the cylinder. The geometry under consideration is of interest from the point of view of generation and transmitting of waves in waveguides, a subject which is of considerable practical importance in microwave engineering and optical fiber communications.
\end{abstract}

DOI: 10.1103/PhysRevAccelBeams.22.040701

\section{INTRODUCTION}

The interfaces between two media with different electromagnetic characteristics give arise to the existence of new types of electromagnetic modes. These surface modes depend on the geometry of the separating boundary and carry an important information on the electromagnetic properties of the contacting media. Among the various types of surface waves, the surface plasmon polaritons (SPPs, for reviews see Refs. [1-4]) have been a powerful tool in the wide range of investigations including surface imaging, surface-enhanced Raman spectroscopy, data storage, biosensors, plasmonic waveguides, photovoltaics, various types of light-emitting devices, plasmonic solar cells, etc. SPPs are evanescent electromagnetic waves propagating along a metal-dielectric interface as a result of collective oscillations of electron subsystem coupled to electromagnetic field. They exist in frequency ranges where the real part of the permittivity undergoes a change of the sign at the interface. Remarkable properties of SPPs include the possibility of concentrating electromagnetic fields beyond the diffraction limit of light waves and enhancing the local field strengths by orders of magnitude [1,2].

Although SPPs are the most thoroughly investigated type of surface polaritons, depending on the dielectric properties

\footnotetext{
*saharian@ysu.am
}

Published by the American Physical Society under the terms of the Creative Commons Attribution 4.0 International license. Further distribution of this work must maintain attribution to the author(s) and the published article's title, journal citation, and DOI. of the active medium (on the use of the terms active and passive media in the physics of surface polaritons see, for instance, Ref. [5]), other forms of surface polaritons may exist. In particular, other materials besides metals, such as semiconductors, organic and inorganic dielectrics, ionic crystals, can support surface polariton type waves. An important direction of recent developments is the extension of plasmonics to the infrared and terahertz ranges of frequencies. This can be done by a suitable choice of the active medium such as doped semiconductors and artificially constructed materials (metamaterials) [6,7].

The techniques used to excite surface polariton modes include prism and grating coupling, strongly focused optical beams, guided photonic modes from another waveguide, electron beams (in particular, in scanning electron microscopes, for reviews see Refs. $[1-3,8])$. Note that the first experimental observation of surface plasmon polaritons was based on measurements of the electron energy loss spectra. From the point of view of an understanding of the fundamental properties and practical applications, of particular interest is the investigation of the effects induced by the curvature of the interface on the generation and propagation of surface polaritons. In the present paper we consider the generation of surface polaritons by a charge circulating around a cylindrical waveguide. Here, the term "surface polariton" is used in the sense clarified, for example, in Ref. [5] (note that, depending on the properties of contacting media, there are also other types of surface waves $[9,10])$. It corresponds to the electromagnetic wave coupled to the induced polarization of the medium. We are interested in the electromagnetic fields and in the intensity of the radiated surface polaritons. 
For a charged particle rotating around a dielectric cylinder, the spectral and angular distribution of the radiation intensity at large distances from the cylinder has been investigated in Refs. [11,12]. It was shown both analytically and numerically that if the Cherenkov condition for permittivity of the cylinder and for the velocity of the particle image on the cylinder surface is obeyed then strong narrow peaks appear in the angular distribution of the radiation intensity on a given harmonic. At these peaks the radiated energy density may exceed the corresponding value for the radiation in the absence of the cylinder by several orders of magnitude. Similar features for the radiation from a charge moving along a helical trajectory around a cylinder have been discussed in Ref. [13]. The radiation of surface waves on the eigenmodes of a dielectric cylinder by a charge circulating around the cylinder is discussed in Ref. [14]. The interference effects between the synchrotron and Smith-Purcell radiations from a charge rotating around a cylindrical grating have been studied in Ref. [15]. In all these investigation it has been assumed that the dielectric functions for both the cylinder and surrounding medium are positive and, hence, the cylinder modes corresponding to surface polaritons are absent. The present paper aims to consider the radiation intensity on those modes.

The organization of the paper is as follows. In the next section we describe the geometry of the problem and present the expression for the azimuthal component of the electric field. The radiation fields for surface polaritons are discussed in Sec. III. By using these fields, in Sec. IV we evaluate the radiation intensity for surface polaritons. Asymptotic limits of the general formula are discussed and the results of numerical analysis are presented. Section V concludes the main results of the paper.

\section{ELECTRIC FIELD OUTSIDE THE CYLINDER}

The system under consideration is a point charge $q$ moving along a circular trajectory of radius $r_{q}$ around a cylinder with radius $r_{c}$ embedded in an outside medium of infinite extent described by dielectric permittivity $\varepsilon_{1}$. The dielectric permittivity of the cylinder will be denoted by $\varepsilon_{0}$ (the magnetic permeabilities for both the cylinder and surrounding medium will be taken to be unit). The geometry of the problem is depicted in Fig. 1. We are interested in the energy losses of the charge in the form of the surface polaritons. The corresponding radiation intensity will be denoted by $I_{(\mathrm{SP})}$. It can be found evaluating the work done by the radiation field on the charged particle:

$$
I_{(\mathrm{SP})}=-\int_{0}^{\infty} d r \int_{0}^{2 \pi} d \phi \int_{-\infty}^{\infty} d z r \mathbf{j} \cdot \mathbf{E}^{(r)},
$$

where $\mathbf{j}$ is the current density for the charge under consideration. The corresponding components in the cylindrical coordinates $(r, \phi, z)$, with the axis $z$ along the cylinder axis, are given by the expression

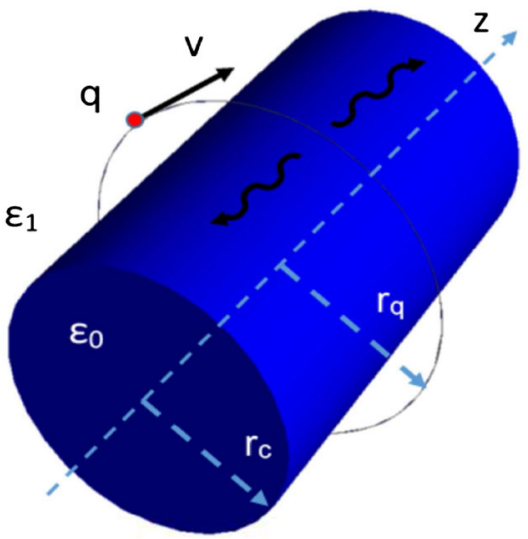

FIG. 1. Geometry of the problem.

$$
j_{l}=\frac{q}{r} v \delta_{l \phi} \delta\left(r-r_{q}\right) \delta\left(\phi-\omega_{0} t\right) \delta(z),
$$

with $v$ and $\omega_{0}=v / r_{q}$ being the velocity and angular velocity of the charge. In (1), $\mathbf{E}^{(r)}$ is the electric field corresponding to surface polaritons. For the problem under consideration the azimuthal component $E_{\phi}^{(r)}$ of the radiation field is required only.

For the electric field $\mathbf{E}(\mathbf{r}, t)$, generated by the source (2), we can write the Fourier expansion

$\mathbf{E}(\mathbf{r}, t)=2 \operatorname{Re}\left[\sum_{n=0}^{\infty} e^{i n \phi-i \omega_{n} t} \int_{-\infty}^{\infty} d k_{z} e^{i k_{z} z} \mathbf{E}_{n}\left(k_{z}, r\right)\right]$,

where $\omega_{n}=n \omega_{0}$ and the prime on the summation sign means that the term $n=0$ should be taken with an additional coefficient $1 / 2$. The representation (3) is obtained from the Fourier expansion of the electric field in the form of the series $\sum_{n=-\infty}^{+\infty}$ by combining the separate contributions from positive and negative $n$ and by using the relations $\mathbf{E}_{n}^{*}\left(k_{z}, r\right)=\mathbf{E}_{-n}\left(-k_{z}, r\right)$ (see also Ref. [16]). The $n=0$ term in Eq. (3) is time independent and will not contribute to the radiation fields. The Fourier components $\mathbf{E}_{n}\left(k_{z}, r\right)$ can be found by using the Green function given in Ref. [11]. Here we will present the expression for the azimuthal component $E_{n \phi}\left(k_{z}, r\right)$, required for the evaluation of the radiation intensity in Eq. (1).

In the region $r>r_{c}$ the Fourier component is decomposed as

$$
E_{n \phi}\left(k_{z}, r\right)=E_{n \phi}^{(0)}\left(k_{z}, r\right)+E_{n \phi}^{(c)}\left(k_{z}, r\right),
$$

where $E_{n \phi}^{(0)}\left(k_{z}, r\right)$ is the corresponding field for a charge rotating in a homogeneous medium with permittivity $\varepsilon_{1}$ and $E_{n \phi}^{(c)}\left(k_{z}, r\right)$ is induced by the presence of the cylinder. For the separate terms in Eq. (4) one has the expressions [for simplicity we omit the arguments $\left.\left(k_{z}, r\right), E_{n \phi}=E_{n \phi}\left(k_{z}, r\right)\right]$ 


$$
\begin{aligned}
E_{n \phi}^{(0)}= & -\frac{q v}{8 \omega_{n} \varepsilon_{1}} \sum_{p, j= \pm 1}\left(j \frac{\omega_{n}^{2}}{c^{2}} \varepsilon_{1}+k_{z}^{2}\right) J_{n+j p}\left(\lambda_{1} r_{q}\right) \\
& \times H_{n+p}\left(\lambda_{1} r\right), \\
E_{n \phi}^{(c)}= & -\frac{i q v}{4 \pi \omega_{n} \varepsilon_{1}} \sum_{p, j= \pm 1}\left(j \frac{\omega_{n}^{2}}{c^{2}} \varepsilon_{1}+k_{z}^{2}\right) B_{n, j p}^{(c)} \\
& \times H_{n+p}\left(\lambda_{1} r\right),
\end{aligned}
$$

where $J_{\nu}(x)$ is the Bessel function, $H_{\nu}(x)=H_{\nu}^{(1)}(x)$ is the Hankel function of the first kind and in the expression for $E_{n \phi}^{(0)}$ it is assumed that $r>r_{q}$. The corresponding expression for $E_{n \phi}^{(0)}$ in the region $r<r_{q}$ is obtained by the replacements $J \rightleftarrows H$. In Eq. (5) we have introduced the notations

$$
\lambda_{j}^{2}=\omega_{n}^{2} \varepsilon_{j} / c^{2}-k_{z}^{2}, \quad j=0,1 .
$$

The coefficients in the expression for the part induced by the cylinder are defined by as

$$
\begin{aligned}
B_{n, p}^{(c)}= & -\frac{\pi}{2 i} H_{n+p}\left(\lambda_{1} r_{q}\right) \frac{V_{n+p}^{J}}{V_{n+p}^{H}}+p \frac{\lambda_{0} J_{n}\left(\lambda_{0} r_{c}\right)}{2 r_{c} \alpha_{n}} \frac{J_{n+p}\left(\lambda_{0} r_{c}\right)}{V_{n+p}^{H}} \\
& \times \sum_{l= \pm 1} \frac{H_{n+l}\left(\lambda_{1} r_{q}\right)}{V_{n+l}^{H}}
\end{aligned}
$$

where $p= \pm 1$,

$$
\alpha_{n}=\frac{\varepsilon_{0}}{\varepsilon_{1}-\varepsilon_{0}}-\frac{\lambda_{0}}{2} J_{n}\left(\lambda_{0} r_{c}\right) \sum_{l= \pm 1} l \frac{H_{n+l}\left(\lambda_{1} r_{c}\right)}{V_{n+l}^{H}},
$$

and for $F=J, H$ we use the notation

$$
V_{n}^{F}=J_{n}\left(\lambda_{0} r_{c}\right) \frac{\partial F_{n}\left(\lambda_{1} r_{c}\right)}{\partial r_{c}}-F_{n}\left(\lambda_{1} r_{c}\right) \frac{\partial J_{n}\left(\lambda_{0} r_{c}\right)}{\partial r_{c}} .
$$

Note that the equation $\alpha_{n}=0$ determines the eigenmodes of the cylinder. By using the properties of cylindrical functions, this equation can be presented in the form given, for example, in Refs. [17,18]. Although the expressions given above are valid for a general case of frequency dependent complex dielectric functions $\varepsilon_{j}=\varepsilon_{j}(\omega), j=0$, 1 , we will ignore the imaginary parts of $\varepsilon_{j}(\omega)$ when describing the eigenmodes.

Radiation fields propagating at large distances from the cylinder, $r \gg r_{q}$, correspond to the integration range in Eq. (3) $k_{z}^{2}<\omega_{n}^{2} \varepsilon_{1} / c^{2}$. For the parts of the fields corresponding to $k_{z}^{2}>\omega_{n}^{2} \varepsilon_{1} / c^{2}$ one has $\lambda_{1}=i\left|\lambda_{1}\right|$ and the Hankel functions with $\lambda_{1}$ in their arguments are reduced to the Macdonald function $K_{\nu}(x)$. For these parts, the fields exponentially decay at large distances from the cylinder. The corresponding modes are divided into two subclasses: guiding modes with $\lambda_{0}^{2}>0$ and surface-type modes with $\lambda_{0}^{2}<0$. In what follows we are interested in the radiation fields for the second subclass of the modes with $k_{z}^{2}>\omega_{n}^{2} \varepsilon_{j} / c^{2}, j=0,1$. The surface polaritons are modes of that type.

\section{RADIATION FIELDS FOR SURFACE-TYPE MODES}

Let us consider the radiation part of the field (3) for large values $|z|$ and for fixed $r$. The problem is symmetric under the replacement $z \rightarrow-z$ and, without loss of generality, we will assume that $z>0$. The exponent in Eq. (3) has no stationary points and the only contribution to the radiation field may come from the poles of the integrand. These poles correspond to the zeros of the function $\alpha_{n}$ in the definition (7) of $B_{n, p}^{(c)}$. For the modes with $\lambda_{j}^{2}<0$ the expression of $\alpha_{n}$ can be rewritten in the form

$$
\alpha_{n}\left(k_{z}\right)=\frac{U_{n}^{(s)}\left(k_{z}\right)}{\left(\varepsilon_{1}-\varepsilon_{0}\right)\left(V_{n}^{(s) 2}-n^{2} u^{(s) 2}\right)},
$$

with the notations

$$
V_{n}^{(s)}=u_{1} \frac{I_{n}^{\prime}}{I_{n}}-u_{0} \frac{K_{n}^{\prime}}{K_{n}}, \quad u^{(s)}=\frac{u_{0}}{u_{1}}-\frac{u_{1}}{u_{0}},
$$

Here and in what follows $I_{n}=I_{n}\left(u_{0}\right), K_{n}=K_{n}\left(u_{1}\right), I_{n}(x)$ is the modified Bessel function, the prime stands for the derivative with respect to the argument of the function and

$$
u_{j}=r_{c} \sqrt{k_{z}^{2}-\omega_{n}^{2} \varepsilon_{j} / c^{2}} .
$$

In Eq. (10), the numerator is defined by the expression

$$
\begin{aligned}
U_{n}^{(s)}\left(k_{z}\right)= & V_{n}^{(s)}\left(\varepsilon_{0} u_{1} \frac{I_{n}^{\prime}}{I_{n}}-\varepsilon_{1} u_{0} \frac{K_{n}^{\prime}}{K_{n}}\right) \\
& -\frac{n^{2} \omega_{n}^{2}}{c^{2}} \frac{r_{c}^{4} k_{z}^{2}}{u_{0}^{2} u_{1}^{2}}\left(\varepsilon_{1}-\varepsilon_{0}\right)^{2} .
\end{aligned}
$$

For the modes under consideration the functions $V_{n+p}^{H}$, $p= \pm 1$, in the denominators of Eq. (7) are presented as

$$
\begin{aligned}
\frac{\pi r_{c}}{2 i} V_{n+p}^{H} & =u_{0} K_{n+p} I_{n+p}^{\prime}-u_{1} I_{n+p} K_{n+p}^{\prime} \\
& =K_{n} I_{n}\left(V_{n}^{(s)}+p n u^{(s)}\right) .
\end{aligned}
$$

From the first equality in Eq. (14) it follows that $-i V_{n+p}^{H}$ is always positive and the functions $V_{n+p}^{H}$ have no zeros. Hence, the only poles correspond to the zeros of $\alpha_{n}$. This feature in the problem related to the guiding modes has 
been already mentioned in Ref. [16]. Note that from Eq. (14) it also follows that $V_{n}^{(s)}>\left|n u^{(s)}\right|$.

In accordance with Eq. (10), the equation for the eigenmodes of the cylinder is reduced to the equation

$$
U_{n}^{(s)}\left(k_{z}\right)=0
$$

Equation (15) coincides with the equation for surface-type modes given, for example, in Refs. [19,20] (on features of propagation and radiation of surface polaritons in cylindrically curved geometries of the interface see, for instance, Refs. [21-28] and references therein). From Eq. (15), as necessary condition for the presence of the surface-type modes with $\lambda_{j}^{2}<0$, we get $\varepsilon_{1} / \varepsilon_{0}<0$. Hence, in order to have surface-type modes the dielectric permittivities of the cylinder and the surrounding medium must have opposite signs. This condition is the same as that for surface-type modes on a planar interface between two media.

In what follows we will assume that $\varepsilon_{1}>0$ and $\varepsilon_{0}<0$. Consequently, the allowed values for $k_{z}$ in the case of surface-type modes are restricted by $k_{z}^{2}>\omega_{n}^{2} \varepsilon_{1} / c^{2}$. The condition $\varepsilon_{0}(\omega)<0$ is realized for example in metals whose plasma frequency $\omega_{p}$ exceeds $\omega$. The simplest model for the corresponding dielectric function is described by the expression

$$
\varepsilon_{0}(\omega)=\varepsilon_{\infty}-\omega_{p}^{2} / \omega^{2},
$$

where $\varepsilon_{\infty}$ is the background dielectric constant. The dispersion described by Eq. (16) is the limiting case of the generalized Drude model (for applications of the Drude type dispersion in surface plasmonics see, for example, Refs. [1,2,6,29]) when the damping coefficient is small. In considering SPPs in the visible and infrared spectral ranges the most commonly used metals are silver, gold, copper, and aluminum. However, it should be noted that in those regions of frequencies the metals are plagued by relatively large ohmic losses which are among the main problems in the performance of plasmonic devices (for a survey of the activities in developing new materials for plasmonics see, for example, Ref. [6]). For the extension of plasmonics to lower frequency ranges, including the $\mathrm{THz}$ range [30], the plasma frequency can be tuned changing the carrier concentrations. One of approaches is to use doped semiconductors. An example is semiconductor indium antimonide (InSb) with the plasma frequency about $1.8 \mathrm{THz}$ and with the background dielectric constant $\varepsilon_{\infty} \approx 15.6$ $[29,31,32]$. Another method is based on the use of artificially constructed materials having given electromagnetic properties (metamaterials).

We will denote by $k_{z}= \pm k_{n, s}, k_{n, s}>0$, the roots of Eq. (15) with respect to $k_{z}$. Here $s$ enumerates the roots for a given $n$. Let us denote by $v_{c}=\omega_{0} r_{c}$ the velocity of the charge image on the cylinder surface. For given $\varepsilon_{j}, n$ and

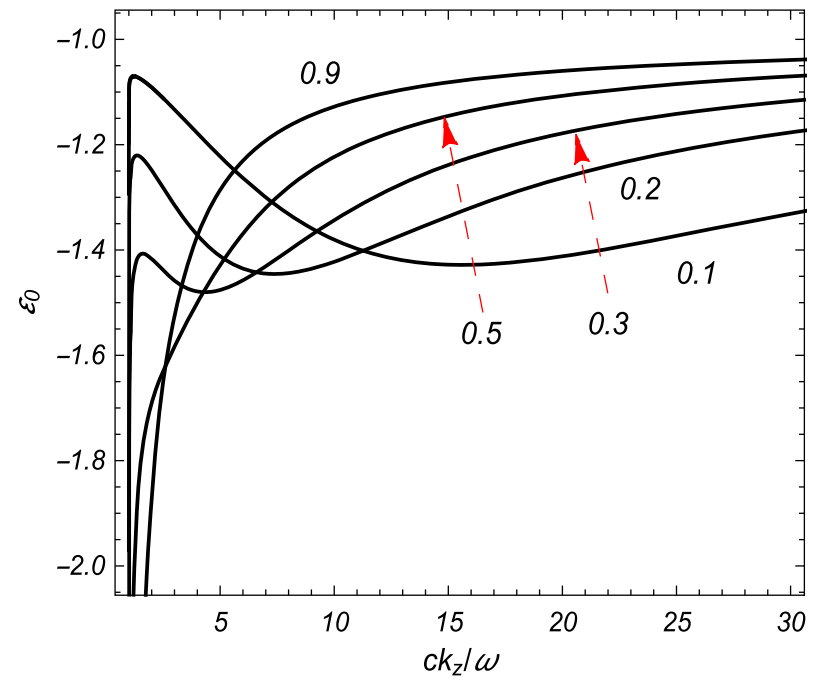

FIG. 2. The roots of the equation $\alpha_{n}=0$ with respect to $\varepsilon_{0}$ versus $c k_{z} / \omega$ for $\varepsilon_{1}=1$ and $n=1$. The numbers near the curves are the values of the ratio $v_{c} / c$.

$v_{c}$, Eq. (15) can be solved with respect to $c k_{z} / \omega$ with $\omega=\omega_{n}$. For fixed $\varepsilon_{1}, n$ and $v_{c}$, we have numerically solved that equation for $c k_{z} / \omega$ as a function of $\varepsilon_{0}$. By taking into account that for a given $\varepsilon_{0}$ one can have several solutions for $c k_{z} / \omega$, in Figs. 2 and 3 we have plotted $\varepsilon_{0}$ versus $c k_{z} / \omega$ for $n=1,2$ and for $\varepsilon_{1}=1$. The numbers near the curves correspond to the values of $v_{c} / c$. For relatively small values of the ratio $v_{c} / c$ there is a region of the values for $\varepsilon_{0}$ where the number of solutions of Eq. (15) is two or three. For large values $c k_{z} / \omega$, corresponding to small wavelengths, $\varepsilon_{0}$ tends to the limiting value $\varepsilon_{0}=-\varepsilon_{1}=-1$. For $n=1$, Eq. (15) has solution for any value of $\varepsilon_{0}<-\varepsilon_{1}$. This is not the case for $n>1$ : there is a critical value $\varepsilon_{0}=\varepsilon_{0 n}$ and Eq. (15) has no solutions for $\varepsilon_{0}<\varepsilon_{0 n}$. For example, for $n=2, v_{c} / c=0.9$ one has $\varepsilon_{0 n}=-3.43$. Note that in

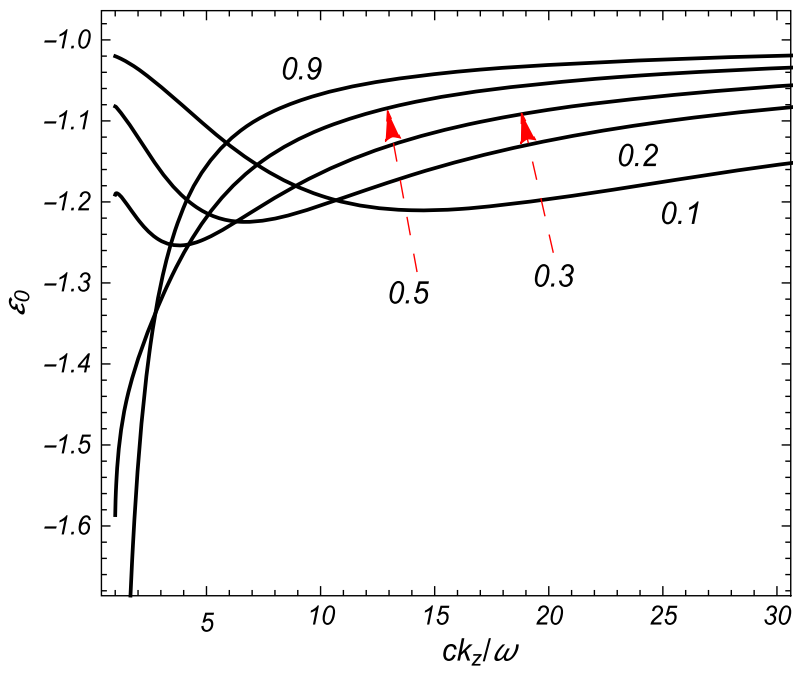

FIG. 3. The same as in Fig. 2 for $n=2$. 
plotting the data of Figs. 2 and 3 no specific form of the dispersion $\varepsilon_{0}=\varepsilon_{0}(\omega)$ is assumed. Given the dispersion law, for example, in the form (16), one can find $k_{n, s}$ for given $n$ and $\omega_{0}$ by solving the equation $\varepsilon_{0}\left(\omega_{n}\right)=$ $f\left(c k_{z} / \omega_{n}\right)$ with respect to $k_{z}$, where the function $\varepsilon_{0}=$ $f\left(c k_{z} / \omega\right)$ is given by the graphs in Figs. 2 and 3. As it is seen from these figures, for surface polaritons the ratio $c k_{z} / \omega$ can be essentially greater than unity. By taking into account that this ratio is also presented as $\lambda_{(0)} / \lambda_{(\mathrm{SP})}$, where $\lambda_{(0)}=2 \pi c / \omega$ is the wavelength of the electromagnetic waves in vacuum and $\lambda_{(\mathrm{SP})}=2 \pi / k_{z}$ is the wavelength of surface polaritons (along the $z$-direction), we see that the wavelength of surface polariton for a given frequency can be essentially smaller than the corresponding wavelength for the electromagnetic radiation in free space. The wide applications of surface polaritons, in particular, are based on this feature.

The asymptotic behavior for large $k_{z}$ can also be seen analytically. By using the asymptotics for the modified Bessel functions for large arguments we can show that for large $u_{j}$ (corresponding to large values of $k_{z}$ ), to the leading order,

$$
\alpha_{n} \approx \frac{\varepsilon_{0}}{\varepsilon_{1}-\varepsilon_{0}}+\frac{u_{0}}{u_{0}+u_{1}} .
$$

The equation $\alpha_{n}=0$ for the eigenmodes of the cylinder is reduced to $\varepsilon_{1} u_{0} \approx-\varepsilon_{0} u_{1}$ with the solution

$$
k_{z}^{2} \approx \frac{\omega_{n}^{2}}{c^{2}} \frac{\varepsilon_{0} \varepsilon_{1}}{\varepsilon_{0}+\varepsilon_{1}} .
$$

This relation coincides with the corresponding relation for a planar interface between two media with permittivities $\varepsilon_{0}$ and $\varepsilon_{1}$. We could expect this feature by taking into account that for small wavelengths (large $k_{z}$ ) the effects of the interface curvature are small. The asymptotic relation (18) shows that $\varepsilon_{0} \rightarrow-\varepsilon_{1}$ for $k_{z} \rightarrow \infty$, the feature seen from Figs. 2 and 3.

For values of $k_{z}$ close to its lowest allowed value for surface polaritons, $\omega_{n} \sqrt{\varepsilon_{1}} / c$, the argument $u_{1}$ of the Macdonald function in the expression for $\alpha_{n}$ is small. By making use of the corresponding asymptotic, for $n=1$ one gets

$$
\alpha_{1} \approx \frac{\varepsilon_{1}}{\varepsilon_{1}-\varepsilon_{0}}+\frac{I_{0}}{2 u_{0} I_{1}} \frac{1}{\ln u_{1}},
$$

where, to the leading order, $u_{0}=\left(\omega_{n} r_{c} / c\right) \sqrt{\varepsilon_{1}+\left|\varepsilon_{0}\right|}$. From Eq. (19) it follows that in the limit under consideration the equation $\alpha_{1}=0$ has solutions for $\varepsilon_{0} \ll-1$ only. For $n>1$ and for $k_{z}$ close to $\omega_{n} \sqrt{\varepsilon_{1}} / c$ we have

$$
\alpha_{n} \approx \frac{1}{2}\left(\frac{\varepsilon_{1}+\varepsilon_{0}}{\varepsilon_{1}-\varepsilon_{0}}+\frac{I_{n}}{I_{n-2}}\right) \text {. }
$$

Based on this relation, it can be seen that for $n>1$ the equation $\alpha_{n}=0$ has no solutions for $\varepsilon_{0} \ll-1$. For $n>1$ there is a critical value $\varepsilon_{0}=\varepsilon_{0 n}$ and the equation $\alpha_{n}=0$ has no solution for $\varepsilon_{0}<\varepsilon_{0 n}$. Again, this feature is confirmed by the numerical data in Fig. 3. For $n>1$ and in the limit $k_{z} \rightarrow \omega_{n} \sqrt{\varepsilon_{1}} / c$ the dielectric function $\varepsilon_{0}$ tends to a finite limiting value $\varepsilon_{0 n}^{(1)}$. For relatively large values of the ratio $v_{c} / c$ this limiting value coincides with the critical value $\varepsilon_{0 n}$. This is not the case for small values of $v_{c} / c$.

Having clarified the properties of the eingemodes for surface polaritons, let us turn to the corresponding radiation fields for the problem at hand. In the presence of the poles $k_{z}= \pm k_{n, s}$, the integration contour in Eq. (3) must be specified. That is done by noting that in physically realistic situations one has $\varepsilon_{j}\left(\omega_{n}\right)=\varepsilon_{j}^{\prime}\left(\omega_{n}\right)+i \varepsilon_{j}^{\prime \prime}\left(\omega_{n}\right)$. By taking into account that $\varepsilon_{j}^{\prime \prime}\left(\omega_{n}\right)>0$ for $\omega_{n}>0$, it can be seen that (see also Ref. $[16,33]$ ) in the integral over $k_{z}$ in Eq. (3) the contour must avoid the poles $k_{z}=k_{n, s}$ from below and the poles $k_{z}=-k_{n, s}$ from above. For the radiation fields in the region $z>0$ we close the integration contour by a semicircle with large radius in the upper complex plane and the integral is expressed in terms of the residues of the integrand. Denoting the radiation part of the electric field by $\mathbf{E}^{(r)}(\mathbf{r}, t)$, we find

$$
\begin{aligned}
\mathbf{E}^{(r)}(\mathbf{r}, t)= & 4 \pi \operatorname{Re}\left\{i \sum_{n=1}^{\infty} e^{i n \phi-i \omega_{n} t}\right. \\
& \left.\times \sum_{s} \operatorname{Res}_{k_{z}=k_{n, s}}\left[e^{i k_{z} z} \mathbf{E}_{n}\left(k_{z}, r\right)\right]\right\} .
\end{aligned}
$$

By using the expressions for the Fourier components $E_{n \phi}\left(k_{z}, r\right)$ from Eq. (5) and evaluating the residue, the azimuthal component of the radiation field outside the cylinder $\left(r>r_{c}\right)$ is presented in the form

$$
\begin{aligned}
E_{\phi}^{(r)}(\mathbf{r}, t)= & \sum_{n=1}^{\infty} \frac{q v r_{c}}{2 \omega_{n} \varepsilon_{1}} \sum_{s} \frac{\lambda_{n, s}^{(0)}}{I_{n} K_{n}^{2} \alpha_{n}^{\prime}\left(k_{n, s}\right)} \sum_{l= \pm 1} \frac{l K_{n+l}\left(\lambda_{n, s}^{(1)} r_{q}\right)}{V_{n}^{(s)}+\ln u^{(s)}} \\
& \times \sum_{p, j= \pm 1} p\left(k_{n, s}^{2}+j \frac{\omega_{n}^{2} \varepsilon_{1}}{c^{2}}\right) \frac{I_{n+j p} K_{n+p}\left(\lambda_{n, s}^{(1)} r\right)}{V_{n}^{(s)}+j p n u^{(s)}} \\
& \times \cos \left(n \phi-\omega_{n} t+k_{n, s} z\right),
\end{aligned}
$$

where $I_{n}=I_{n}\left(\lambda_{n, s}^{(0)} r_{c}\right), K_{n}=K_{n}\left(\lambda_{n, s}^{(1)} r_{c}\right)$, and

$$
\lambda_{n, s}^{(j)}=\left|\lambda_{j}\left(k_{n, s}\right)\right|=\sqrt{k_{n, s}^{2}-\omega_{n}^{2} \varepsilon_{j} / c^{2}} .
$$

Here, $V_{n}^{(s)}$ and $u^{(s)}$ are defined by Eq. (11) with $u_{j}=r_{c} \lambda_{n, s}^{(j)}$. The part in Eq. (4) corresponding to the field in a homogeneous medium does not contribute to the radiation field (22). 
Note that the expression (22) for the azimuthal component of the electric field corresponding to the radiated surface-type modes is valid for all values of $z>0$ (the expression for the field in the region $z<0$ is obtained by changing the sign of $k_{n, s} z$ in the argument of the cosine function). This follows from the fact that the contribution of the poles $k_{z}=k_{n, s}$ to the integral over $k_{z}$ in Eq. (3) remains the same for all values of $z>0$. For large values of $z$ and for a fixed $r$ close to the cylinder surface the contribution of (4) dominates in the total electric field, whereas for $z$ close to the charge it is mixed with other contributions (proper field of the charge and the field corresponding to the radiation at large distances from the cylinder). In deriving Eq. (22) we have assumed that $z>0$. The expression in the right-hand side of Eq. (22) is an analytic function of $z$ in that region and can be analytically continued to the value $z=0$.

\section{RADIATION INTENSITY}

Substituting the radiation field (21) in Eq. (1), the radiation intensity is presented as the sum of the intensities on separate harmonics with a given $n, I_{(\mathrm{SP})}=\sum_{n=1}^{\infty} I_{(\mathrm{SP}) n}$, where

$$
\begin{aligned}
I_{(\mathrm{SP}) n}= & -\frac{q^{2} v^{2}}{2 \omega_{n} \varepsilon_{1}} \sum_{s} \frac{r_{c} \lambda_{n, s}^{(0)}}{\alpha_{n}^{\prime}\left(k_{n, s}\right) I_{n} K_{n}^{2}} \sum_{l= \pm 1} \frac{l K_{n+l}\left(\lambda_{n, s}^{(1)} r_{q}\right)}{V_{n}^{(s)}+\ln u^{(s)}} \\
& \times \sum_{p, j= \pm 1} p\left(k_{n, s}^{2}+j \frac{\omega_{n}^{2} \varepsilon_{1}}{c^{2}}\right) \frac{I_{n+j p} K_{n+p}\left(\lambda_{n, s}^{(1)} r_{q}\right)}{V_{n}^{(s)}+j p n u^{(s)}} .
\end{aligned}
$$

This expression can be further simplified by using the relation

$$
\begin{aligned}
& \sum_{p, j= \pm 1} p\left(k_{n, s}^{2}+j \frac{\omega_{n}^{2} \varepsilon_{1}}{c^{2}}\right) \frac{I_{n+j p} K_{n+p}(x)}{V_{n}^{(s)}+j p n u^{(s)}} \\
& =\frac{2 I_{n}}{\lambda_{n, s}^{(1)} u^{(s)}} \frac{\omega_{n}^{2} \varepsilon_{1}}{c^{2}}\left(\lambda_{n, s}^{(0)} \frac{I_{n}^{\prime}}{I_{n}}-\lambda_{n, s}^{(1)} \frac{K_{n}^{\prime}}{K_{n}}\right) \sum_{p} \frac{p K_{n+p}(x)}{V_{n}^{(s)}+p n u^{(s)}} .
\end{aligned}
$$

The radiation intensity is presented as

$$
\begin{aligned}
I_{(\mathrm{SP}) n}= & -\frac{q^{2} v^{2} r_{c}}{\omega_{n}\left(\varepsilon_{1}-\varepsilon_{0}\right)} \sum_{s} \frac{\lambda_{n, s}^{(0) 2}}{\alpha_{n}^{\prime}\left(k_{n, s}\right) K_{n}^{2}} \\
& \times\left[\sum_{l= \pm 1} \frac{l K_{n+l}\left(\lambda_{n, s}^{(1)} r_{q}\right)}{V_{n}^{(s)}+\ln u^{(s)}}\right]^{2}\left(\lambda_{n, s}^{(0)} \frac{I_{n}^{\prime}}{I_{n}}-\lambda_{n, s}^{(1)} \frac{K_{n}^{\prime}}{K_{n}}\right) .
\end{aligned}
$$

For a given angular velocity $\omega_{0}$ of the charge rotation, the radius of the orbit appears in the argument of the functions $K_{n+l}\left(\lambda_{n, s}^{(1)} r_{q}\right)$ and through $v^{2}$ in the numerator of the first factor in the right-hand side. For distances from the cylinder surface, $r_{q}-r_{c}$, much larger that the radiation wavelength the radiation intensity is suppressed by the factor $\exp \left[-2 \lambda_{n, s}^{(1)}\left(r_{q}-r_{c}\right)\right]$.

In order to further clarify the dependence of the radiation intensity on the parameters of the problem let us consider some limiting cases. As it has been shown above, for values of $\varepsilon_{0}$ close to $-\varepsilon_{1}$ the roots $k_{n, s}$ are large. By using the asymptotic expressions of the modified Bessel functions for large values of the arguments, to the leading order we get

$$
I_{(\mathrm{SP}) n} \approx \frac{4 q^{2} v^{2} \varepsilon_{0}}{\left(\varepsilon_{1}^{2}-\varepsilon_{0}^{2}\right) r_{q}^{3} \omega_{n}} \exp \left[-2 \frac{\omega_{n}}{c} \frac{\varepsilon_{1}\left(r_{q}-r_{c}\right)}{\sqrt{-\varepsilon_{0}-\varepsilon_{1}}}\right] .
$$

This shows that the radiation intensity tends to zero in the limit $\varepsilon_{0} \rightarrow-\varepsilon_{1}$. For $\varepsilon_{0} \ll-\varepsilon_{1}$ the surface polariton type modes are present for the main harmonic $n=1$ only. By taking into account that in this range the roots for $k_{z}$ are close to the limiting value $\omega_{n} \sqrt{\varepsilon_{1}} / c$ and the arguments of the Macdonald functions in Eq. (26) are small, to the leading order we find

$$
I_{(\mathrm{SP}) 1} \approx \frac{q^{2} c\left|\varepsilon_{0}\right|}{2 \varepsilon_{1}^{5 / 2} r_{q}^{2}}\left(r_{q}^{2} / r_{c}^{2}-1\right)^{2} \exp \left(-\frac{c \sqrt{\left|\varepsilon_{0}\right|}}{\varepsilon_{1} v_{c}}\right) .
$$

This shows that the radiation intensity, as a function of the dielectric permittivity of the cylinder, vanishes in the limit $\varepsilon_{0} \rightarrow-\infty$. For $n>1$ and in the limit $k_{z} \rightarrow \omega_{n} \sqrt{\varepsilon_{1}} / c$, the dielectric permittivity of the cylinder, determined from the mode equation for surface polaritons, tends to a finite limiting value, $\varepsilon_{0} \rightarrow \varepsilon_{0 n}^{(1)}$. Consequently, the quantity $\lambda_{n, s}^{(0)}$ in Eq. (26) tends to the finite limit $\left(\omega_{n} / c\right) \sqrt{\varepsilon_{1}+\left|\varepsilon_{0 n}^{(1)}\right|}$. In the limit under consideration the arguments of the Macdonald functions in Eq. (26) are small. By using the corresponding asymptotic expressions, it can be seen that the radiation intensity $I_{(\mathrm{SP}) n}$ approaches a finite limiting value.

In the numerical analysis for the radiation intensity it is convenient to introduce a new variable $z_{n}=c k_{z} / \omega_{n}$. In terms of the latter one has $u_{j}=n\left(v_{c} / c\right) \sqrt{z_{n}^{2}-\varepsilon_{j}}$. For a given harmonic $n$, the root $z_{n}$ is a function of $v_{c} / c, \varepsilon_{0}$ and $\varepsilon_{1}$, determined from Eq. (15). Examples of the dependence of $z_{n}$ on $\varepsilon_{0}$, for $\varepsilon_{1}=1$ and for several values of $v_{c} / c$, are presented in Figs. 2 and 3. For surface polaritons one has $\varepsilon_{1} \leq c k_{z} / \omega<\infty$. In Fig. 4 we have plotted the number of the radiated quanta in the form of surface polaritons on a given harmonic $n$ per period of the charge rotation $T=2 \pi / \omega_{0}$,

$$
N_{(\mathrm{SP}) n}=T \frac{I_{(\mathrm{SP}) n}}{\hbar \omega_{n}},
$$




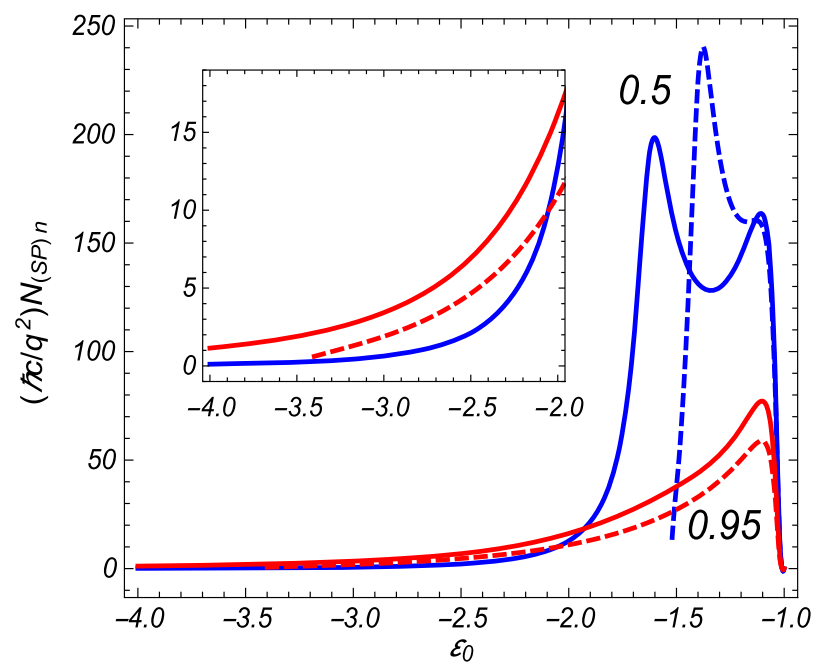

FIG. 4. The number of the radiated quanta in the form of surface polaritons versus the dielectric permittivity of the cylinder. The graphs are plotted for $v / c=0.5,0.95$ (numbers near the curves) and for $n=1,2$ (full and dashed curves, respectively).

as a function of $\varepsilon_{0}$ for $\varepsilon_{1}=1, r_{c} / r_{q}=0.95$. The numbers near the curves correspond to the values of $v / c$ and the full (dashed) curves correspond to $n=1 \quad(n=2)$. For the critical values of the cylinder dielectric permittivity in the case $n=2$ one has $\varepsilon_{0 n} \approx-1.52$ for $v / c=0.5$ and $\varepsilon_{0 n} \approx-3.44$ for $v / c=0.9$.

In Fig. 5 we have plotted the dependence of the number of the radiated surface polaritons on the dielectric permittivity of the cylinder for $v / c=0.5$ and for higher harmonics $n=3,5,10$ (numbers near the curves). Again we see the presence of critical values of the dielectric permittivity for the radiation of surface polaritons on the harmonics with $n>1$. The critical value increases with increasing $n$. For $n=3,5,10$ one has $\varepsilon_{0 n} \approx-1.37$,

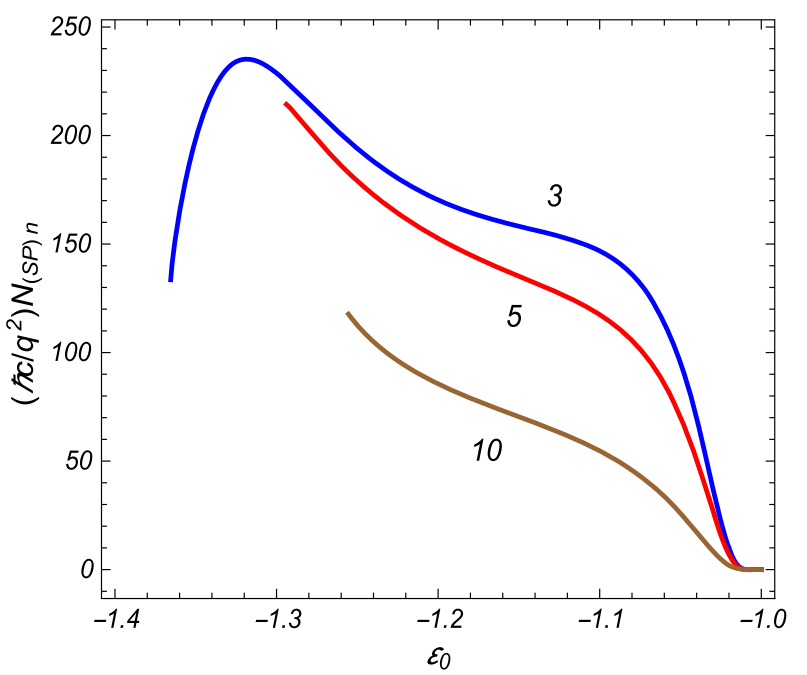

FIG. 5. The same as in Fig. 3 for $v / c=0.5$ and $n=3,5,10$ (numbers near the curves).
$-1.29,-1.26$, respectively. In these cases the critical values $\varepsilon_{0 n}$ coincide with the limiting values $\varepsilon_{0 n}^{(1)}$.

It is of interest to compare the radiation intensity for surface polaritons with the radiation for guiding modes $\left(\lambda_{1}^{2}<0<\lambda_{0}^{2}\right)$ from a charge rotating around a cylinder having dielectric permittivity $\varepsilon_{0}>0$ in a given spectral range. The intensity for the part of the corresponding radiation propagating in the region outside the cylinder has been investigated in Ref. [14] (the radiation of guiding modes by a charge circulating inside the dielectric cylinder has been discussed in Ref. [34]). The numerical results were presented for a cylinder made of quartz. Note that for guiding modes, with increasing harmonic number $n$ the number of roots $k_{n, s}$ increases for some critical values of the harmonic. For the example considered in Ref. [14], the number of roots was 1 for $1 \leq n \leq 7,2$ for $8 \leq n \leq 12$, and 3 for $13 \leq n \leq 16$. For the corresponding number of the quanta radiated on the guiding modes per period of the rotation by an electron of energy $2 \mathrm{MeV}$ and for $r_{c} / r_{q}=0.99$ ones has $N_{(\mathrm{GM}) n}<0.25 q^{2} /(\hbar c)$. The numerical data for these values of the energy and the ratio $r_{c} / r_{q}$, similar to those depicted in Fig. 4, show that $\mathrm{f}$ or $-5<\varepsilon_{0}<0$ the number of the radiated surface polaritons is essentially larger. For example, in the case of $n=1$ one gets $N_{(\mathrm{SP}) n} \approx 4.23 q^{2} /(\hbar c)$ for $\varepsilon_{0}=-3$ and $N_{(\mathrm{SP}) n} \approx 45.49 q^{2} /(\hbar c)$ for $\varepsilon_{0}=-1.5$.

By using the approach described above, we have also evaluated the total radiation intensity, $I_{(\mathrm{G}) n}$, for the same geometry depicted in Fig. 1 and for a cylinder with positive dielectric permittivity $\varepsilon_{0}>0$ (the corresponding results will be presented elsewhere). For comparison with the radiation of surface polaritons, in Fig. 6 we present the analog of Fig. 4 for $N_{(\mathrm{G}) n}=T I_{(\mathrm{G}) n} /\left(\hbar \omega_{n}\right)$. The graphs are

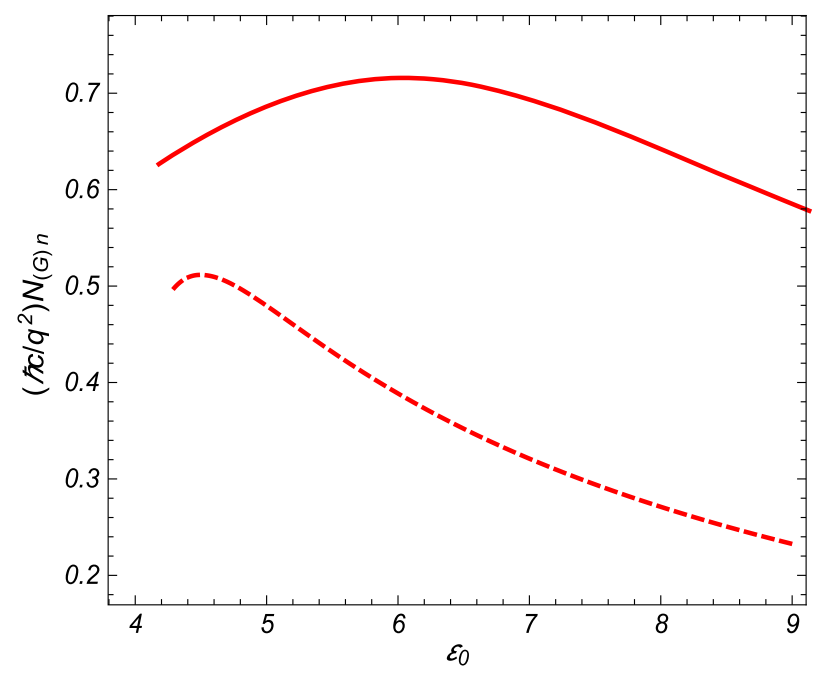

FIG. 6. The number of the radiated quanta for guiding modes, as a function of the dielectric permittivity of the cylinder, for $v / c=0.95$ and for $n=1,2$ (full and dashed curves). 
plotted for $\varepsilon_{1}=1, v / c=0.95, r_{c} / r_{q}=0.95, n=1$ (full curve) and $n=2$ (dashed curve). For $v / c=0.5$ and for the same values of the remaining parameters there are no guiding modes for $\varepsilon_{0}<15$ and the corresponding radiation is absent. Again, we see that in the considered ranges of the dielectric permittivity of the cylinder the radiation intensity of surface polaritons is essentially larger than the one for guiding modes. Similar to the case of surface polaritons, for the examples presented in Fig. 6 there is a critical value $\varepsilon_{0 n}$ for the dielectric permittivity of the cylinder with no guiding modes in the range $\varepsilon_{0}<\varepsilon_{0 n}$. For graphs in Fig. 6 one has $\varepsilon_{0 n}=4.18$ for $n=1$ and $\varepsilon_{0 n}=4.30$ for $n=2$. The truncation of the graphs is related to the existence of these critical values.

\section{SUMMARY}

Cylindrical waveguides are essential components in photonic integrated circuits. They have also attracted much interest due to potential applications in communications technology and in the microcavity lasers. In the present paper we have investigated the radiation of surface polaritons by a charge rotating around a dielectric cylinder with permittivity $\varepsilon_{0}$. For the corresponding waves the component of the wave vector along the cylinder axis obey the condition $k_{z}^{2}>\omega_{n}^{2} \varepsilon_{1} / c^{2}$ and they are radiated on the eigenmodes of the cylinder, determined by the zeros of the function $\alpha_{n}\left(k_{z}\right)$ for a given harmonic. For the existence of solutions to this equation the dielectric permittivities for the cylinder and surrounding medium should have opposite signs. We have considered the case $\varepsilon_{0}<0<\varepsilon_{1}$. The radiation intensity for surface waves on a given harmonic $n$ is given by Eq. (26). For the main harmonic $n=1$, the surface polaritons are radiated for all values of the cylinder dielectric function in the range $\varepsilon_{0}<-\varepsilon_{1}$. For higher harmonics $n>1$, there exists a critical value $\varepsilon_{0 n}$ with the absence of surface polariton radiation in the range $\varepsilon_{0}<-\varepsilon_{0 n}$. The radiation wavelength increases with approaching $\varepsilon_{0}$ to the limiting value $-\varepsilon_{1}$. In that range, the wavelength of surface polaritons is much smaller that the wavelength of electromagnetic radiation in free space with the same frequency. We have also demonstrated that, for a given harmonic, the number of radiated quanta for surface polaritons can be essentially larger than that for guiding modes of the cylinder.

Note that in the consideration above we have not fixed the absolute values of the cylinder and the charge orbit radii. The features described were sensitive on the ratio of these quantities and, in dependence of the orbit radius, they are valid in different spectral ranges. For a given energy of the charge, the spectral range of the radiation is determined by the radius of the rotation orbit. Depending on that range, various materials for the cylinder can be used: silver, gold, copper, aluminum in the visible and infrared spectral ranges and appropriately doped semiconductors and metamaterials in the $\mathrm{THz}$ range. For example, let us consider the radiation of surface polaritons corresponding to the local peak in Fig. 4 closer to the limiting value $-\varepsilon_{1}=-1$ for $n=1$ and $v / c=0.5$. For that peak one has $c k_{z} / \omega_{n} \approx 20$ which means that the radiation wavelength for surface polaritons is about 20 times smaller than the wavelength of corresponding free space electromagnetic radiation. For a cylindrical waveguide with the radius of the order of $1 \mathrm{~mm}$, the wavelength of surface polaritons is in the submillimeter range that corresponds to the $\mathrm{THz}$ frequency range of free space electromagnetic radiation. The corresponding circular motion for electron can be generated by laboratory magnetic fields (for other configurations of external fields in a more general case of a helical trajectory see also [16]).

We have considered an idealized problem with an infinite length cylindrical waveguide. The corresponding results well approximate real situations when the length of the dielectric cylinder is much larger than the radius of the rotation orbit and the wavelength of the radiated polaritons. For a cylinder with finite length, in the corresponding electrodynamical problem additional boundary conditions must be imposed at the ends. An interesting effect that arises at the termination is the transformation of surface polaritons to free electromagnetic field propagating in surrounding medium (radiation of electromagnetic waves by surface polaritons). A similar effect of conversion of the energy from surface polaritons to free radiation takes place in bent waveguides $[23,35]$. The corresponding electromagnetic radiation can be used for the investigation of properties of surface polaritons [36].

For the problem under consideration, in addition to the radiation of surface polaritons, there is also radiation propagating at large distances from the cylinder. The latter corresponds to synchrotron radiation modified by the presence of the cylinder. For the respective electromagnetic fields one has $k_{z}^{2}<\omega_{n}^{2} \varepsilon_{1} / c^{2}$ and their radial dependence is given by the Hankel functions $H_{n+p}\left(\lambda_{1} r\right)$ with positive $\lambda_{1}$. The features of synchrotron radiation for a cylinder made of material with negative dielectric permittivity will be discussed in a separate paper.

\section{ACKNOWLEDGMENTS}

V. Kh. K. is grateful to Prof. Michael Berry for discussions related to the subject of the present paper. A. A. S. has been supported by Grant No. 18T-1C397 from the Science Committee of the Ministry of Education and Science of the Republic of Armenia.

\section{APPENDIX: TOTAL RADIATION INTENSITY}

In this Appendix we consider the total radiation intensity evaluated as

$$
I=-\int_{0}^{\infty} d r \int_{0}^{2 \pi} d \phi \int_{-\infty}^{\infty} d z r \mathbf{j} \cdot \mathbf{E} .
$$


By using the expression (2) for the current density and the Fourier expansion (3), it is presented in the form

$$
I=-2 q v \lim _{r \rightarrow r_{q}} \sum_{n=1}^{\infty} \operatorname{Re}\left[\int_{-\infty}^{\infty} d k_{z} E_{n \phi}\left(k_{z}, r\right)\right],
$$

where we keep in the integrand $r>r_{q}$ for the convenience of the further evaluation. As it has been argued above, in Eq. (A2), the poles $k_{z}=k_{n, s}\left(k_{z}=-k_{n, s}\right)$ in the integration range $\left|k_{z}\right|>\omega_{n} \sqrt{\varepsilon_{1}} / c$ are avoided by small semicircles $C_{n, s}^{+}(\rho)\left[C_{n, s}^{-}(\rho)\right]$ with radius $\rho$ in the lower (upper) halfplane of the complex plane $k_{z}$.

By using the expression for $E_{n \phi}\left(k_{z}, r\right)$ [see Eqs. (4) and (5)], it can be seen that for real $k_{z}$ and in the region $\left|k_{z}\right|>\omega_{n} \sqrt{\varepsilon_{1}} / c$ the Fourier component $E_{n \phi}\left(k_{z}, r\right)$ is purely imaginary. Hence, in that region the only nonzero contribution to the integral in Eq. (A2) comes from the integrals over the semicircles $C_{n, s}^{ \pm}(\rho)$. In the limit $\rho \rightarrow 0$ the sum of these integrals is combined as the residue at $k_{z}=k_{n, s}$ :

$$
\sum_{j=+,-} \int_{C_{n, s}^{j}(\rho)} d k_{z} E_{\phi n}\left(k_{z}, r\right)=2 \pi i \operatorname{Res}_{k_{z}=k_{n, s}}\left[E_{\phi n}\left(k_{z}, r\right)\right] .
$$

The contribution of this part of the integral to Eq. (A2) coincides with the radiation intensity $I_{(\mathrm{SP})}$ for surface polaritons, given by Eq. (24) for a given harmonic. As a result, the total radiation intensity is presented as

$I=I_{(\mathrm{SP})}-2 q v \lim _{r \rightarrow r_{q}} \sum_{n=1}^{\infty} \operatorname{Re}\left[\int_{-\omega_{n} \sqrt{\varepsilon_{1}} / c}^{\omega_{n} \sqrt{\varepsilon_{1}} / c} d k_{z} E_{n \phi}\left(k_{z}, r\right)\right]$.

For the second term in the right-hand side of Eq. (A4) one has $\lambda_{1}^{2}>0$ and it corresponds to the radiation observed at large distances from the cylinder. In particular, it can be seen that the part with $E_{n \phi}^{(0)}\left(k_{z}, r\right)$ from Eq. (5) coincides with the radiation intensity for the synchrotron radiation in a homogeneous medium with permittivity $\varepsilon_{1}$ (see, for instance, [37]).

[1] V. M. Agranovich and D. L. Mills, Surface Polaritons: Electromagnetic Waves at Surfaces and Interfaces (NorthHolland, Amsterdam, 1982).

[2] S. A. Maier, Plasmonics: Fundamentals and Applications (Springer, New York, 2007).

[3] Plasmonics: From Basics to Advanced Topics, edited by S. Enoch and N. Bonod (Springer, New York, 2012).

[4] M. I. Stockman et al., Roadmap on plasmonics, J. Opt. 20, 043001 (2018).

[5] K. Welford, Surface plasmon-polaritons and their uses, Opt. Quantum Electron. 23, 1 (1991).
[6] P. R. West, S. Ishii, G. V. Naik, N. K. Emani, V. M. Shalaev, and A. Boltasseva, Searching for better plasmonic materials, Laser Photonics Rev. 4, 795 (2010).

[7] N. C. Lindquist, P. Nagpal, K. M. McPeak, D. J. Norris, and S.-H. Oh, Engineering metallic nanostructures for plasmonics and nanophotonics, Rep. Prog. Phys. 75, 036501 (2012).

[8] Zh. Han and S. I. Bozhevolnyi, Radiation guiding with surface plasmon polaritons, Rep. Prog. Phys. 76, 016402 (2013).

[9] J. A. Polo, Jr. and A. Lakhtakia, Surface electromagnetic waves: A review, Laser Photonics Rev. 5, 234 (2011).

[10] T. K. Sarkar, M. N. Abdallah, M. Salazar-Palma, and W. M. Dyab, Surface Plasmons-Polaritons, Surface Waves, and Zenneck Waves: Clarification of the terms and a description of the concepts and their evolution, IEEE Trans. Antennas Propag. 59, 77 (2017).

[11] L. Sh. Grigoryan, A. S. Kotanjyan, and A. A. Saharian, Electromagnetic field Green function in cylindrically symmetric inhomogeneous medium, Izv. Nats. Akad. Nauk Arm., Fiz. 30, 239 (1995) [Sov. J. Contemp. Phys. 30, 1 (1995)].

[12] A. S. Kotanjyan, H. F. Khachatryan, A. V. Petrosyan, and A. A. Saharian, On features of radiation from charged particle rotating around a dielectric cylinder, Izv. Nats. Akad. Nauk Arm., Fiz. 35, (2000) [Sov. J. Contemp. Phys. 35, 1 (2000)].

[13] A. A. Saharian and A. S. Kotanjyan, Synchrotron radiation from a charge moving along a helix around a dielectric cylinder, J. Phys. A 42, 135402 (2009).

[14] A. S. Kotanjyan, A. R. Mkrtchyan, A. A. Saharian, and V. Kh. Kotanjyan, Radiation of surface waves from a charge rotating around a dielectric cylinder, J. Instrum. 13, C01016 (2018).

[15] A. A. Saharian, A. S. Kotanjyan, A. R. Mkrtchyan, and B. V. Khachatryan, Synchrotron and Smith-Purcell radiations from a charge rotating around a cylindrical grating, Nucl. Instrum. Methods Phys. Res., Sect. B 402, 162 (2017).

[16] A. A. Saharian, A. S. Kotanjyan, and M. L. Grigoryan, Electromagnetic field generated by a charge moving along a helical orbit inside a dielectric cylinder, J. Phys. A 40, 1405 (2007).

[17] J. A. Stratton, Electromagnetic theory (McGraw-Hill, New York, 1941).

[18] J. D. Jackson, Classical Electrodynamics (Wiley, New York, 1999).

[19] J. C. Ashley and L. C. Emerson, Dispersion relations for non-radiative surface plasmons on cylinders, Surf. Sci. 41, 615 (1974).

[20] H. Khosravi, D. R. Tilley, and R. Loudon, Surface polaritons in cylindrical optical fibers, J. Opt. Soc. Am. A 8, 112 (1991).

[21] M. V. Berry, Attenuation and focusing of electromagnetic surface waves rounding gentle bends, J. Phys. A 8, 1952 (1975).

[22] U. Schröter and A. Dereux, Surface plasmon polaritons on metal cylinders with dielectric core, Phys. Rev. B 64, 125420 (2001). 
[23] K. Hasegawa, J. U. Nöckel, and M. Deutsch, Curvatureinduced radiation of surface plasmon polaritons propagating around bends, Phys. Rev. A 75, 063816 (2007).

[24] J.-W. Liaw and P.-T. Wu, Dispersion relation of surface plasmon wave propagating along a curved metal-dielectric interface, Opt. Express 16, 4945 (2008).

[25] M. Guasoni, Analytical approximations of the dispersion relation of the plasmonic modes propagating around a curved dielectric-metal interface, J. Opt. Soc. Am. B 28, 1396 (2011).

[26] J. Polanco, R. Fitzgerald, and A. Maradudin, Propagation of p-polarized surface plasmon polaritons circumferentially around a locally cylindrical surface, Opt. Commun. 316, 120 (2014).

[27] I. A. Kotelnikov and G. V. Stupakov, Electromagnetic surface waves on a conducting cylinder, Phys. Lett. A 379, 1187 (2015).

[28] M. V. Berry, Surface waves with high angular momentum: leakage from remote caustics, and tightly coiled streamlines, Eur. J. Phys. 39, 045807 (2018).

[29] T. H. Isaac, J. Gómez Rivas, J. R. Sambles, W. L. Barnes, and E. Hendry, Surface plasmon mediated transmission of subwavelength slits at THz frequencies, Phys. Rev. B 77, 113411 (2008).
[30] D. M. Mittleman, Frontiers in terahertz sources and plasmonics, Nat. Photonics 7, 666 (2013).

[31] S.C. Howells and L. A. Schlie, Transient terahertz reflection spectroscopy of undoped $\mathrm{InSb}$ from 0.1 to 1.1 THz, Appl. Phys. Lett. 69, 550 (1996).

[32] Sh. Lin, K. Bhattarai, J. Zhou, and D. Talbayev, Giant $\mathrm{THz}$ surface plasmon polariton induced by high-index dielectric metasurface, Sci. Rep. 7, 9876 (2017).

[33] A. S. Kotanjyan and A. A. Saharian, Radiation from a charge circulating inside a waveguide with dielectric filling, Mod. Phys. Lett. A 17, 1323 (2002).

[34] A. A. Saharian and A. S. Kotanjyan, Synchrotron radiation inside a dielectric cylinder, Int. J. Mod. Phys. B 26, 1250033 (2012).

[35] W. Wang, Q. Yang, F. Fan, H. Xu, and Z. L. Wang, Light propagation in curved silver nanowire plasmonic waveguides, Nano Lett. 11, 1603 (2011).

[36] A. Drezet, A. Hohenau, D. Koller, A. Stepanov, H. Ditlbacher, B. Steinberger, F. R. Aussenegg, A. Leitner, and J.R. Krenn, Leakage radiation microscopy of surface plasmon polaritons, Mater. Sci. Eng. B 149, 220 (2008).

[37] A. A. Sokolov and I. M. Ternov, Radiation from Relativistic Electrons (ATP, New York, 1986). 